\title{
Cone-beam computed tomography findings of impacted upper canines
}

\author{
Ludmilla Mota da Silva Santos ${ }^{1, *}$, Luana Costa Bastos ${ }^{2}$, Christiano Oliveira-Santos ${ }^{3}$, \\ Silvio José Albergaria da Silva ${ }^{4}$, Frederico Sampaio Neves ${ }^{5}$, Paulo Sérgio Flores Campos ${ }^{2}$ \\ ${ }^{1}$ Department of Endodontics, Araçatuba Dental School, Paulista State University, Araçatuba, SP, Brazil \\ ${ }^{2}$ Department of Oral Radiology, School of Dentistry, Federal University of Bahia, Salvador, BA, Brazil \\ ${ }^{3}$ Department of Stomatology, Oral Public Health, and Forensic Dentistry, School of Dentistry, University of São Paulo, \\ Ribeirão Preto, SP, Brazil \\ ${ }^{4}$ Department of Endodontics, School of Dentistry, Federal University of Bahia, Salvador, BA, Brazil \\ ${ }^{5}$ Department of Oral Diagnosis, Piracicaba Dental School, State University of Campinas, Piracicaba, SP, Brazil
}

\section{ABSTRACT}

Purpose: To describe the features of impacted upper canines and their relationship with adjacent structures through three-dimensional cone-beam computed tomography (CBCT) images.

Materials and Methods: Using the CBCT scans of 79 upper impacted canines, we evaluated the following parameters: gender, unilateral/bilateral occurrence, location, presence and degree of root resorption of adjacent teeth (mild, moderate, or severe), root dilaceration, dental follicle width, and presence of other associated local conditions.

Results: Most of the impacted canines were observed in females (56 cases), unilaterally (51 cases), and at a palatine location (53 cases). Root resorption in adjacent teeth and root dilaceration were observed in 55 and 47 impacted canines, respectively. In most of the cases, the width of the dental follicle of the canine was normal; it was abnormally wide in 20 cases. A statistically significant association was observed for all variables, except for root dilaceration $(p=0.115)$ and the side of impaction $(p=0.260)$.

Conclusion: Root resorption of adjacent teeth was present in most cases of canine impaction, mostly affecting adjacent lateral incisors to a mild degree. A wide dental follicle of impacted canines was not associated with a higher incidence of external root resorption of adjacent teeth. (Imaging Sci Dent 2014; 44: 287-92)

KEY WORDS: Cone-Beam Computed Tomography; Root Resorption; Cuspid; Tooth, Impacted

\section{Introduction}

Canines play a key role in facial aesthetics, development of the dental arch, and occlusion. Disturbances in the eruption of permanent upper canines are relatively common because these canines develop deep within the jaw and follow a longer path to erupt into the oral cavity when compared to the other teeth. Treatment of impacted canines is often challenging and may involve different specialties,

Received May 15, 2014; Revised July 1, 2014; Accepted July 15, 2014

*Correspondence to : Dr. Ludmilla Mota da Silva Santos

Department of Endodontics, School of Dentistry, Federal University of Bahia, Avenida Araújo Pinho, 72, Canela, Zip code: 40110-912, Salvador, BA, Brazil

Tel) 55-71-3283-8964, Fax) 55-71-3270-8512, E-mail) ludmillasantos@yahoo.com.br such as surgery and orthodontics. With early detection, timely interception, and correct orthodontic treatment and surgery, impacted canines may erupt and correctly locate in the dental arch. ${ }^{1}$

Non-erupted canines are the second most common group to suffer impaction, following only the third molars. The etiology of non-erupted canines is multifactorial and remains unclear. ${ }^{2}$ However, the lack of space in the dental arch, ${ }^{3}$ prolonged retention of deciduous canines, ${ }^{4}$ absence of adjacent lateral incisors, ${ }^{5}$ root dilaceration, and ankylosis of permanent canines are the most common local factors associated with the impaction of maxillary canines. In general, canines are more often impacted in the palatal position than in the buccal position, ${ }^{6}$ except in the Chinese 
population which seems to be more prone to canine impaction in the buccal position. ${ }^{7}$ The role of genetic factors in the occurrence of impacted canines has also been pointed out. $^{3}$

The impaction of canines may cause the migration of adjacent teeth, loss of arch length and width, and occasional cystic lesions, tumors, and infections. However, an important consequence of the abnormal path of canine eruption within the dental alveolar process is the resorption of adjacent teeth roots, compromising the longevity of these teeth. ${ }^{8}$ This process is often asymptomatic; therefore, when the resorption is clinically diagnosed, the process may be so advanced that it no longer allows for any type of conservative treatment. ${ }^{9}$

Although new diagnostic imaging techniques have been introduced in recent years, conventional two-dimensional (2D) radiographs, including panoramic, occlusal, and periapical radiographs remain the most commonly used modality for the primary diagnosis and localization of non-erupted canines, evaluation, and treatment planning. ${ }^{10}$ However, $2 \mathrm{D}$ radiographic images may display the presence of nonerupted teeth but are very limited in demonstrating the exact location of these teeth, the impact on neighboring teeth and other adjacent structures, and the anatomy of roots, which are information of absolute relevance in treatment planning. ${ }^{11}$

Computed tomography (CT) eliminates image superimposition and allows reconstruction of scanned structures in different planes, as well as three-dimensional (3D) reconstructions. ${ }^{11,12}$ Another recent and increasingly available modality in dentistry, cone-beam computed tomography (CBCT) produces high-quality diagnostic 3D images with minimal distortion, relatively low cost, and significantly reduced radiation dose as compared to other CT modalities. ${ }^{13}$ Due to its increasing availability, there has been a significant current interest of researchers and clinicians in revisiting different clinical conditions using CBCT, which has allowed significant advances in dental practice. ${ }^{14}$

In cases of impacted canines, images obtained by CBCT are accurate in determining their buccal-palatal location and angulation; they are also useful in determining the proximity of impacted canines to the roots of adjacent incisors and premolars, as well as the degree of resorption. .,15,16 $^{-16}$ These features are important in treatment planning to move the canine in the arch and decrease the risk of root resorption of adjacent teeth. Various types of imaging software allow CBCT image reconstructions in multiple planes, providing a better view of the canine position. ${ }^{17}$

Given the importance of correct diagnosis through imag- ing exams for a safer therapeutic approach, and considering the use of CBCT for the assessment of impacted teeth, this study aimed to characterize the main features of impacted upper canines and their effect on adjacent structures by using CBCT imaging.

\section{Materials and Methods}

The present study was carried out following approval from the local Ethics and Research Committee (Protocol number: CAAE 05297712.0.0000.5024), and informed consent was obtained from all volunteers. The sample consisted of 67 individuals (80 impacted canines) who underwent CBCT examination performed for the evaluation of impacted maxillary canines previously detected by panoramic and/or periapical radiographs. CBCT images were obtained between August 2009 and September 2011 with a KODAK 3D K9000 CBCT unit (Kodak Dental Systems, Carestream, Rochester, NY, USA). No restriction related to age or gender was required. Impactions caused by osseous lesions and patients with systemic bone disease

Table 1. Relationship between impacted canines and variables assessed in this study.

\begin{tabular}{|c|c|c|c|}
\hline Variables & $\mathrm{n}=79$ & $\%$ & $p$ value \\
\hline Gender & & & 0.000 \\
\hline Female & 56 & 70.9 & \\
\hline Male & 23 & 29.1 & \\
\hline Type of occurrence & & & 0.013 \\
\hline Unilateral & 51 & 64.6 & \\
\hline Bilateral & 28 & 35.4 & \\
\hline Side & & & 0.260 \\
\hline Right & 34 & 43 & \\
\hline Left & 45 & 57 & \\
\hline Localization & & & 0.000 \\
\hline Buccal & 17 & 21.5 & \\
\hline Palatine & 53 & 67.1 & \\
\hline Central & 9 & 11.4 & \\
\hline Root resorption & & & 0.001 \\
\hline Presence & 55 & 69.6 & \\
\hline Absence & 24 & 30.4 & \\
\hline Root dilaceration & & & 0.115 \\
\hline Presence & 47 & 59.5 & \\
\hline Absence & 32 & 40.5 & \\
\hline Dental follicle width & & & 0.000 \\
\hline Normal & 59 & 74.3 & \\
\hline Wide & 20 & 25.3 & \\
\hline \multicolumn{4}{|l|}{ Other associated local conditions } \\
\hline Retained deciduous canines & 27 & 34.2 & 0.007 \\
\hline $\begin{array}{l}\text { Transposition between canine } \\
\text { and premolar }\end{array}$ & 2 & 2.5 & 0.000 \\
\hline Supernumerary tooth & 2 & 2.5 & 0.000 \\
\hline Odontoma & 1 & 1.3 & 0.000 \\
\hline Enamel and/or dentin defects & 7 & 8.9 & 0.000 \\
\hline
\end{tabular}



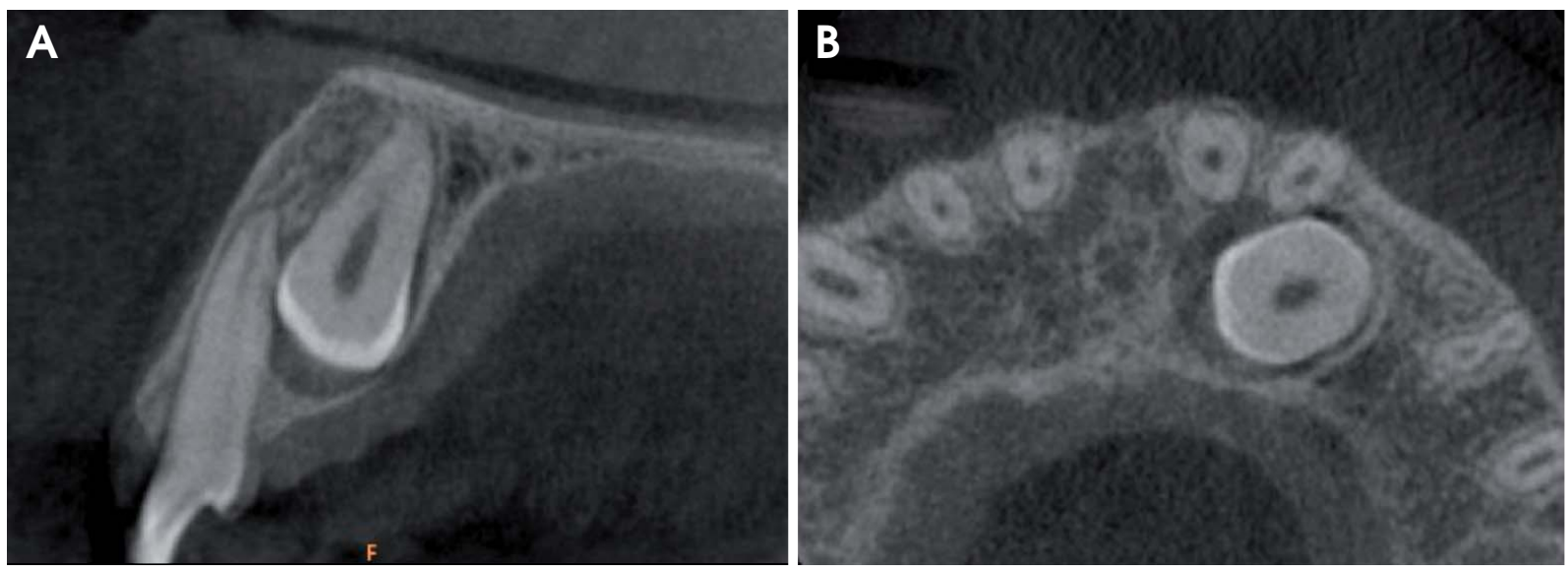

Fig. 1. Sagittal (A) and axial (B) slices of cone-beam computed tomography (CBCT) show an impacted canine causing mild root resorption of the lateral incisor.

were excluded from the study.

An impacted tooth is one that fails to erupt into the dental arch within a specific time period. In this study, a tooth was considered impacted when it was completely or partially intraosseous and had more than $2 / 3$ of its root developed. One tooth was excluded from the sample due to the presence of a dentigerous cyst. The final sample consisted of 79 impacted canines from 66 individuals. Information on gender, unilateral/bilateral occurrence, side, location, root dilaceration, dental follicle width of the impacted canines, root resorption of the adjacent teeth, and the other associated local conditions observed is presented in Table 1.

The images were evaluated in all three tomographic planes (axial, sagittal, and coronal) and in the 3D reconstruction in a dimmed light room, on a $22^{\prime \prime}$ flat screen monitor (Dell Precision $390^{\mathrm{TM}}$, Dell Inc., Round Rock, Texas, USA) by a single oral radiologist with more than six years of experience of CBCT using the KDIS3D CBCT image software (version 2.1.11, Kodak Dental Systems, Carestream Health, Rochester, NY, USA).

The age and the gender of each patient were recorded. Using the images obtained from CBCT, we analyzed the following parameters: 1. unilateral or bilateral impaction; 2. buccal, palatal, or central location; 3. root resorption of adjacent teeth (central incisor, lateral incisor, and first premolar); 4. root anatomy: presence or absence of dilaceration; 5 . width of the dental follicle: normal $(\leq 2 \mathrm{~mm})$ or wider than normal $(>2 \mathrm{~mm})$; 6 . other associated local conditions: retention of the corresponding deciduous canine, transposition of the permanent canine, presence of supernumerary teeth or odontomas, and enamel and/or dentin defects. Among them, root resorption was determined by the classification proposed by Ericson and Kurol: $\left.{ }^{18} 1\right)$ absence of resorption (intact root surface, but a compromised layer of cementum), 2) mild (resorption to half the thickness of dentin) (Fig. 1), 3) moderate (resorption very close to the pulp, without exposure of pulpal tissue), and 4) severe (pulp exposed due to resorption).

Data were analyzed using SAS software 9.1 (SAS Institute, Cary, NC, USA). Descriptive analysis for demographic data (absolute and relative frequencies) and the $\chi^{2}$ or Fisher's exact test were performed. The significance level was set at $5 \%$.

\section{Results}

The subjects' ages ranged from 12 to 55 years (mean age: 22 years). Most of the impacted canines were observed in females (56 cases), unilaterally (51 cases), and at a palatine location (53 cases). Root resorption in adjacent teeth and root dilaceration were observed in 55 and 47 impacted canines, respectively. In most of the cases, the width of the dental follicle of the canine was normal; it was abnormally wide in 20 cases. In the case of other associated local conditions, we observed that retained deciduous canines ( 27 cases) and the presence of enamel and/or dentine defects in the impacted canines ( 7 cases) were the most common. A statistically significant association was observed with all variables $(p<0.05)$, except for the root dilaceration $(p=0.115)$ and the side of impaction $(p=0.260)$.

In total, 55 canines $(69.6 \%)$ caused resorption in 71 adjacent teeth. The root resorption was associated with lateral incisors (48 cases), central incisors (19 cases), and premolars (4 cases). A relatively high occurrence of mild and moderate root resorption was observed. Severe resorption was only observed in lateral incisors (6 cases) (Table 2). 
Table 2. Occurrence of root resorption on adjacent teeth and its degree of severity.

\begin{tabular}{ccc}
\hline Teeth & $\mathrm{n}=71$ & $\%$ \\
\hline Central incisor & 19 & 26.8 \\
Mild & 13 & 68.4 \\
Moderate & 6 & 31.6 \\
Severe & 0 & 0 \\
Lateral incisor & 48 & 67.6 \\
Mild & 33 & 68.8 \\
Moderate & 6 & 12.5 \\
Severe & 9 & 18.7 \\
Premolar & 4 & 5.6 \\
Mild & 3 & 75 \\
Moderate & 1 & 25 \\
Severe & 0 & 0 \\
\hline
\end{tabular}

Impacted canines were more frequently located palatally in both males and females. Females presented impacted canines at a central location in only $7.1 \%$ of the cases, while males, in $21 \%$ of the cases. However, this difference was not statistically significant $(p=0.054)$. Root resorption was present in nearly $70 \%$ of the cases, and the frequency was similar for both males and females $(p=0.995)$. An association between the presence of root resorption of adjacent teeth and the location of the impacted canine was also not statistically significant $(p=0.204)$ (Fig. 2). However, impacted canines with an increased width of the dental follicle were associated with a relatively low frequency of root resorption on adjacent teeth; this was statistically significant ( $p=0.027$ ) (Fig. 3).

\section{Discussion}

Comparative studies between 2D and 3D images have demonstrated superiority of the latter in identifying aspects related to impacted maxillary canines, particularly the location and adjacent root resorption of permanent teeth, which are crucial in developing the right treatment plan for the patient. ${ }^{17}$

Our results regarding the gender distribution, type of occurrence, and localization of impacted canines resemble those found in the literature, ${ }^{6,15,19-21}$ although some studies showed a tendency towards buccal impactions. ${ }^{7,15}$ The association between gender and the location of impacted canines was not significant, although women presented palatally impacted canines more often. The location of impacted canines appears to be related to genetic traits or has geographic differences. ${ }^{6}$ This could be attributed to the differences in jawbone structure for different ethnicities, like the shape of the maxillary arch, the height of the palate,

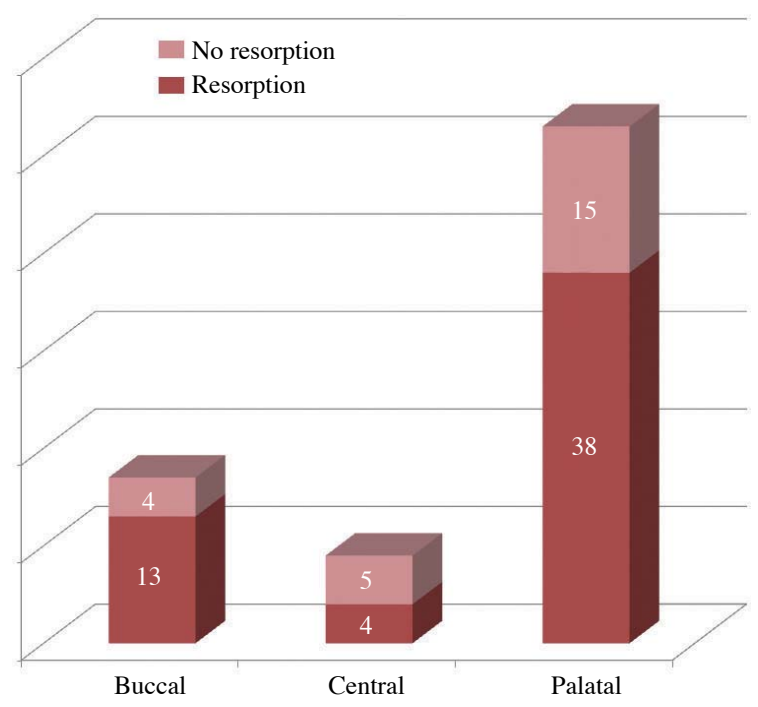

Fig. 2. Distribution of adjacent teeth presenting resorption according to the location of the impacted canines.

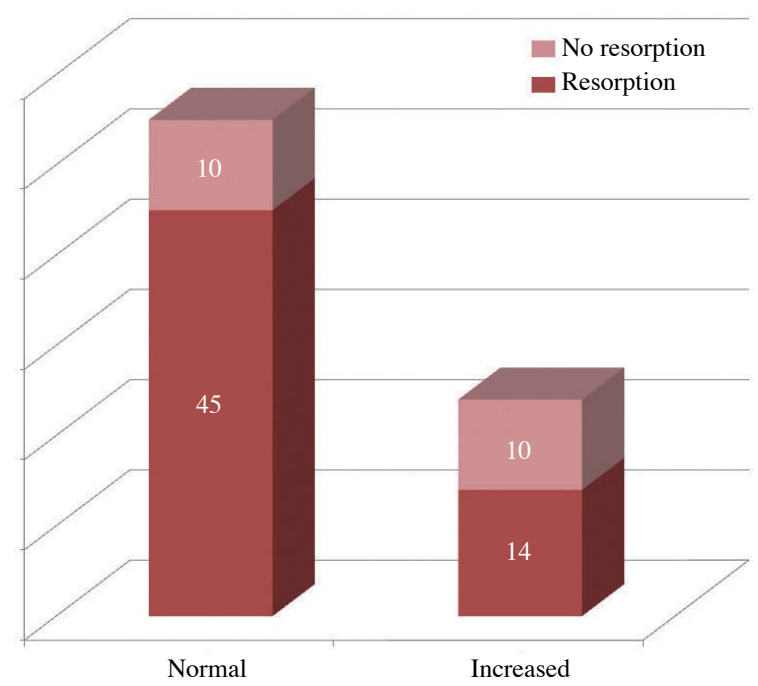

Fig. 3. Distribution of adjacent teeth presenting resorption according to the follicle width of the impacted canines (i.e., normal or increased).

and the nasal cavity, which influence the jaw shape and cause variations in the position of the tooth germ in the arch. Furthermore, craniofacial growth differences influence the amount of space in the dental arch. However, it must be emphasized that some studies applied panoramic radiographs for this evaluation, ${ }^{20,22}$ which did not provide a complete visualization of the positioning of impacted teeth without other exams.

Impacted canines are important factors related to the external root resorption of adjacent teeth. In this study, the prevalence of lateral and central incisor root resorption by 
impacted canines was similar to the literature ${ }^{6,15,23}$ Root resorption of the first premolar is uncommon and represents an important event that is often misdiagnosed or diagnosed late, when the tooth has suffered resorption that cannot be controlled, leading to its extraction. ${ }^{24}$ Gender does not seem to influence root resorption. Conversely, the location of the canine in the dental arch seems to play an important role in adjacent root resorption, particularly when the canine is in the palate. Location of the tooth was also described as an important factor elsewhere. ${ }^{25}$ The results of the present study demonstrated that most resorptive processes may be detected using CBCT, because the images provide 3D information, while conventional radiographs are limited in detecting lesions on the buccal and palatine aspects. $^{26}$

Root dilaceration was present in most impacted canines. Root shape-particularly in cases where there is pronounced dilaceration - can impact the clinical approach of impacted teeth. Dilacerations can be accurately diagnosed by CBCT, in both subtle and pronounced cases. For subtle dilacerations, CBCT can visualize the direction and interaction with neighboring teeth, while for the pronounced cases, there is a possibility for visualizing the anatomical aspects of the root and its interactions with the bone, sinus region, and palatal or buccal anatomical structures, which is favorable for treatment planning.

The dental follicle thickness was within the normal range in most cases; this is similar to the findings of other studies. ${ }^{6,15}$ The association between the occurrence of root resorption and the thickness of the dental follicle was significant. However, resorption seems to occur irrespective of the size of the dental follicle. ${ }^{6,27}$ The resorption of the neighboring permanent teeth during the eruption of the maxillary canine was most likely an effect of the adjacent active pressure during the eruption and the cellular activities in tissues at the contact points, which are part of the eruptive mechanisms. Asymmetrical dental follicles are more common in ectopic canines and are more accurately identified through CBCT.

Deciduous canines were retained in less than half the cases. ${ }^{6}$ This retention can hinder the eruption of the permanent canines; therefore, their extraction is indicated, although the success rate for spontaneous eruption appears to be low in cases wherein the patient reached adolescence at the beginning of the treatment. ${ }^{28}$ Prevalence of transposition observed between the impacted canine and the first premolar was very small which corroborated the relative rarity of this anomaly in most populations. ${ }^{29}$ It is known that the most common transposition involves canines and the first premolars ${ }^{30}$ and occurs when the path of eruption of the maxillary canines is diverted by genetic and/or environmental factors. ${ }^{31}$

The treatment of transposition between the canine and the first premolar depends on the stage of tooth development at the time of the diagnosis of the anomaly. These teeth should remain transposed when the roots are fully developed. ${ }^{32}$ The presence of supernumerary teeth and odontomas associated with impacted canines was observed in a few cases. In fact, the occurrence of supernumerary teeth in the region of the lateral incisor and the upper canine is not rare, ${ }^{33}$ and although they are usually asymptomatic, the extraction of supernumerary teeth and odontomas is an elective treatment. ${ }^{34}$

CBCT should not be used routinely for the assessment of unerupted teeth in the context of a root resorption diagnosis, but it may be indicated when conventional intraoral radiography does not supply adequate information. ${ }^{35} \mathrm{CBCT}$ images of the impacted maxillary canines can determine with precision the presence or absence of a tooth; the follicle width; the inclination of the long axis of the teeth; the relative buccal, central, and palatal positions; the $3 \mathrm{D}$ proximity and resorption of the roots of the adjacent teeth; the local anatomic considerations; and finally, the overall stage of dental development. ${ }^{36}$ Therefore, we agreed on a reliable assessment of the 3D position and on the improved localization and surgical-orthodontic management of maxillary impacted canines using CBCT.

In conclusion, root resorption of adjacent teeth is present in most cases of canine impaction, mostly affecting the adjacent lateral incisors to a mild degree. In addition, the increased dental follicles of the impacted canines were not associated with the relatively high incidence of the external root resorption of adjacent teeth. Further, there was no relationship between impacted canines with root dilaceration and the side of impaction.

\section{References}

1. Bedoya MM, Park JH. A review of the diagnosis and management of impacted maxillary canines. J Am Dent Assoc 2009; 140: 1485-93.

2. Cooke J, Wang HL. Canine impactions: incidence and management. Int J Periodontics Restorative Dent 2006; 26: 483-91.

3. Peck S, Peck L, Kataja M. The palatally displaced canine as a dental anomaly of genetic origin. Angle Orthod 1994; 64: 24956.

4. Baccetti T. Risk indicators and interceptive treatment alternatives for palatally displaced canines. Semin Orthod 2010; 16 : 186-92.

5. Becker A. In defense of the guidance theory of palatal canine 
displacement. Angle Orthod 1995; 65: 95-8.

6. Walker L, Enciso R, Mah J. Three-dimensional localization of maxillary canines with cone-beam computed tomography. Am J Orthod Dentofacial Orthop 2005; 128: 418-23.

7. Sajnani A, King N. Dental age of children and adolescents with impacted maxillary canines. J Orofac Orthop. 2012; 73: 359-64.

8. Westphalen VP, Gomes de Moraes I, Westphalen FH, Martins WD, Souza PH. Conventional and digital radiographic methods in the detection of simulated external root resorptions: a comparative study. Dentomaxillofac Radiol 2004; 33: 233-5.

9. Zhong YL, Zeng XL, Jia QL, Zhang WL, Chen L. Clinical investigation of impacted maxillary canine. Zhonghua Kou Qiang Yi Xue Za Zhi 2006; 41: 483-5.

10. Chalakkal P, Thomas AM, Chopra S. Displacement, location, and angulation of unerupted permanent maxillary canines and absence of canine bulge in children. Am J Orthod Dentofacial Orthop 2011; 139: 345-50.

11. Mah JK, Alexandroni S. Cone-beam computed tomography in the management of impacted canines. Semin Orthod 2010; 16: 199-204.

12. Agrawal JM, Agrawal MS, Nanjannawar LG, Parushetti AD. CBCT in orthodontics: the wave of future. J Contemp Dent Pract 2013; 14: 153-7.

13. Scarfe WC, Farman AG, Sukovic P. Clinical applications of cone-beam computed tomography in dental practice. J Can Dent Assoc 2006; 72: 75-80.

14. Chen Y, Duan P, Meng Y, Chen Y. Three-dimensional spiral computed tomographic imaging: a new approach to the diagnosis and treatment planning of impacted teeth. Am J Orthod Dentofacial Orthop 2006; 130: 112-6.

15. Liu DG, Zhang WL, Zhang ZY, Wu YT, Ma XC. Localization of impacted maxillary canines and observation of adjacent incisor resorption with cone-beam computed tomography. Oral Surg Oral Med Oral Pathol Oral Radiol Endod 2008; 105: 91-8.

16. Alqerban A, Jacobs R, Fieuws S, Nackaerts O, SEDENTEXCT Project Consortium, Willems G. Comparison of 6 cone-beam computed tomography systems for image quality and detection of simulated canine impaction-induced external root resorption in maxillary lateral incisors. Am J Orthod Dentofacial Orthop 2011; 140: e129-39.

17. Bjerklin K, Ericson S. How a computerized tomography examination changed the treatment plans of 80 children with retained and ectopically positioned maxillary canines. Angle Orthod 2006; 76: 43-51.

18. Ericson S, Kurol PJ. Resorption of incisors after ectopic eruption of maxillary canines: a CT study. Angle Orthod 2000; 70: 415-23.

19. Rozylo-Kalinowska I, Kolasa-Raczka A, Kalinowski P. Dental age in patients with impacted maxillary canines related to the position of the impacted teeth. Eur J Orthod 2011; 33: 492-7.

20. Katsnelson A, Flick WG, Susarla S, Tartakovsky JV, Miloro M. Use of panoramic $\mathrm{x}$-ray to determine position of impacted maxillary canines. J Oral Maxillofac Surg 2010; 68: 996-1000.

21. Chung DD, Weisberg M, Pagala M. Incidence and effects of genetic factors on canine impaction in an isolated Jewish population Am J Orthod Dentofacial Orthop 2011; 139: e331-5.

22. Leuzinger M, Dudic A, Giannopoulou C, Kiliaridis S. Rootcontact evaluation by panoramic radiography and cone-beam computed tomography of super-high resolution. Am J Orthod Dentofacial Orthop 2010; 137: 389-92.

23. Siegel R, Stós W, Dyras M, Urbanik A, Wojciechowski W, Sztuk S. Assessment of degree and extent of resorption of incisor roots adjacent to impacted maxillary canines. Przegl Lek 2010; 67: 268-74.

24. Kim Y, Hyun HK, Jang KT. The position of maxillary canine impactions and the influenced factors to adjacent root resorption in the Korean population. Eur J Orthod 2011; 34: 302-6.

25. Postlethwaite KM. Resorption of premolar roots by ectopic canines Br Dent J 1989; 167: 397-8.

26. da Silveira HL, Silveira HE, Liedke GS, Lermen CA, Dos Santos RB, de Figueiredo JA. Diagnostic ability of computed tomography to evaluate external root resorption in vitro. Dentomaxillofac Radiol 2007; 36: 393-6.

27. Smailienè D, Sidlauskas A, Lopatienè K, Guzevičienè V, Juodžbalys G. Factors affecting self-eruption of displaced permanent maxillary canines. Medicina (Kaunas) 2011; 47: 163-9.

28. Ericson S, Bjerklin K, Falahat B. Does the canine dental follicle cause resorption of permanent incisor roots? A computed tomographic study of erupting maxillary canines. Angle Orthod 2002; 72: 95-104.

29. Burnett SE. Prevalence of maxillary canine-first premolar transposition in a composite African sample. Angle Orthod 1999; 69: 187-9.

30. Ely NJ, Sherriff M, Cobourne MT. Dental transposition as a disorder of genetic origin. Eur J Orthod 2006; 28: 145-51.

31. Synodinos PN, Polyzois I. Maxillary canine-first premolar transposition in the permanent dentition: treatment considerations and a case report. J Ir Dent Assoc 2010; 56: 264-7.

32. Halazonetis DJ. Horizontally impacted maxillary premolar and bilateral canine transposition. Am J Orthod Dentofacial Orthop 2009; 135: 380-9.

33. Liu DG, Zhang WL, Zhang ZY, Wu YT, Ma XC. Three-dimensional evaluations of supernumerary teeth using cone-beam computed tomography for 487 cases. Oral Surg Oral Med Oral Pathol Oral Radiol Endod 2007; 103: 403-11.

34. Nagaraj K, Upadhyay M, Yadav S. Impacted maxillary central incisor, canine, and second molar with 2 supernumerary teeth and an odontoma Am J Orthod Dentofacial Orthop 2009; 135: 390-9.

35. European Commission [Internet]. Radiation protection No 172: cone beam CT for dental and maxillofacial radiology (evidencebased guidelines). 2012 [cited 2014 May 14]. Available from: http://www.sedentexct.eu/files/radiation_protection_172.pdf.

36. Rossini G, Cavallini C, Cassetta M, Galluccio G, Barbato E. Localization of impacted maxillary canines using cone beam computed tomography. Review of the literature. Ann Stomatol (Roma) 2012; 3: 14-8. 